\title{
El tratamiento quirúrgico sería más eficaz que el tratamiento conservador en espondilolistesis lumbar degenerativa
}

Surgical treatment seems better than conservative treatment in lumbar degenerative spondylolisthesis

Weinstein J et al. N Engl J Med $2007 ; 356: 2257-70$

\section{Objetivo}

Comparar la eficacia del tratamiento quirúrgico (Q) y la del tratamiento conservador (NoQ) en pacientes con espóndilolistesis degenerativa lumbar (EDL).

\section{Diseño}

Cohorte con un seguimiento promedio de dos años, con un subgrupo de individuos asignados a una intervención aleatorizada.

\section{Lugar}

13 centros de Estados Unidos.

\section{Pacientes}

Se hizo una selección aleatoria de 607 individuos con más de 12 semanas de claudicación neurogénica y radiculopatía, con signos neurológicos asociados, sin respuesta al tratamiento conservador y estenosis lumbar en el contexto de EDL. Trescientos cuatro pacientes fueron asignados a un grupo donde se los volvió a aleatorizar a tratamiento $Q \circ N o Q$ y los 303 restantes acordaron el tratamiento a través de una conversación con su médico. Fueron excluidos aquellos con espondilolisis y espondilolistesis ístmica.

\section{Intervención}

Q: laminectomía descompresiva estándar, con o sin artrodesis y con o sin instrumentación transpedicular.

NoQ: disminución de las actividades diarias, rehabilitación kinesiológica, educación para la realización de ejercicios en el hogar, bloqueos peridurales y antiinflamatorios no esteroides.

\section{Medición de resultados principales}

Cuestionarios SF-36 y Oswestry Disability Index (ODI). Ambos con puntajes de 0 a 100, correspondiendo la puntuación más alta a menor sintomatología y/o mayor funcionalidad en el primero y a la inversa en el segundo. El análisis fue por intención de tratar*.

\section{Resultados principales}

Se resumen en la tabla 1.

Tabla 1: diferencias a favor de los resultados en el grupo "cirugía", comparándolo con el grupo "tratamiento conservador" en pacientes con espóndilolistesis degenerativa lumbar.

\begin{tabular}{|c|c|c|c|c|}
\hline \multirow{2}{*}{\multicolumn{2}{|c|}{ Instrumento }} & \multicolumn{3}{|c|}{ Diferencia de puntos e IC95\% } \\
\hline & & Tres meses & Un aĩo & Dos aĩos \\
\hline \multirow[t]{2}{*}{ SF-36 } & Dolor & $17,8(14,9$ a 20,6$)$ & $18,8(15,5$ a 22,1$)$ & $18,1(14,5$ a 21,7$)$ \\
\hline & Funcionalidad & $13,9(11,1$ a 16,8$)$ & $19,4(16,1$ a 22,7$)$ & $18,3(14,6$ a 21,9$)$ \\
\hline \multicolumn{2}{|c|}{ Oswestry } & $-14,6(-16,8$ a 12,4 & $-17,9 \quad(-20,5$ a $-15,3$ & $-16,7 \quad(-19,5$ a $\quad-13,9)$ \\
\hline
\end{tabular}

El $10 \%$ de los pacientes sometidos a $Q$ sufrieron un desgarro dural y $12 \%$ fueron reoperados a los dos años.

\section{Conclusiones}

Hasta los dos años de seguimiento, los pacientes con estenosis espinal y ELD tratados $Q$ mostraron una significativa mejoría del dolor y la función física que los sometidos a tratamiento NoQ.

Palabras clave: espondilolistesis lumbar degenerativa, cirugía, tratamiento conservador.

Key words: lumbar degenerative spondylolisthesis, surgical treatment, conservative treatment.

Fuente de Financiamiento: Musculoeskeletal foundation, Medtronic, Synthes, Stryker, De Puy Spine, AOSpine, K2M.

\section{Comentario}

La EDL es común en pacientes de edad avanzada, asociándose frecuentemente con canal estrecho lumbar'. Los pacientes suelen consultar por dolor lumbar y claudicación de la marcha y dado que se trata de un trastorno progresivo, no se ven restringidos en forma aguda para deambular. Del mismo modo, la topografía donde se desarrolla esta entidad (habitualmente L4-L5, L3-L4) no provoca síntomas neurológicos de neurona motora superior. De este modo, el tratamiento conservador es la primera línea de tratamiento, experimentando muchos pacientes un alivio de los síntomas. Sin embargo, y por tratarse de un problema de alteración de la relación entre el continente y el contenido dentro del canal raquídeo, la cirugía descompresiva muchas veces termina siendo el tratamiento final ${ }^{2,3,4}$. El objetivo del artículo que comentamos es novedoso ya que no existían trabajos comparativos de esta índole en la literatura actual ${ }^{5}$.

Vale destacar que muchos de los pacientes incluidos en esta investigación se beneficiaron con el tratamiento conservador. Sin embargo, cuando se los comparó con mediciones validadas como el SF-36 y ODI, la cirugía fue más beneficiosa, por lo menos hasta los dos años de seguimiento. Entre sus limitaciones mencionamos la escasa aceptación por parte de los pacientes a la aleatorización y la heterogeneidad de variantes de tratamiento en el grupo observacional.

\section{Conclusiones del comentador}

Es muy importante para un buen resultado clínico una adecuada selección de los pacientes. Muchos pueden beneficiarse con tratamiento conservador, y muchos otros pueden con el quirúrgico. Se debe discutir con el paciente las distintas opciones terapéuticas mencionando su efectividad y sus potenciales efectos adversos.

Marcelo Valacco [ Instituto de Ortopedia y Traumatología "Prof. Dr. Carlos E. Ottolenghi”, Patología Espinal, Hospital Italiano de Buenos Aires. marcelo.valacco@ hospitalitaliano.org.ar ]

Recibido el 02/04/08 y aceptado el 04/08/08

alacco M. El tratamiento quirúrgico sería más eficaz que el tratamiento conservador en espondilolistesis. Evid. actual. práct. ambul; 11(4):106, JulAgo.2008 . Comentado de: Weinstein J y col. Surgical versus nonsurgical treatment for lumbar degenerative spondylolisthesis. $\mathrm{N}$ Engl $\mathrm{J}$ Med. 2007 May 31; 356 (22):2257-70. PMID: 17538085. Disponible en URL: http://content.nejm.org/cgi/reprint/356/22/2257.pdf (último acceso 04/08/08).

Referencia

1. Herkowitz H. Spine update. Degenerative lumbar spondylolisthesis. Spine. 1995 May 1;20(9):1084-90.

2. Vibert B y col. Treatment of instability and spondylolisthesis: surgical versus nonsurgical treatment. Clin Orthop Relat Res. 2006 Feb; 443:222-7.

3. Knaub M y col. Lumbar spinal stenosis: indications for arthrodesis and spinal instrumentation. Instr Course Lect 2005; 54: 313-9.

4. Fischgrund $\mathrm{J}$ y col. Volvo Award winner in clinical studies. Degenerative lumbar spondylolisthesis with spinal stenosis: a prospective, randomized study comparing decompressive laminectomy and arthrodesis with and without spinal instrumentation. Spine. 1997 Dec 15:22(24):2807-12.

5. Sengupta D y col. Degenerative spondylolisthesis: review of current trends and controversies. Spine. 2005 Mar 15;30(6 Suppl):S71-81. 